\title{
Contribuições da iniciação à docência na formação em saúde através das vivências nos espaços extramuros na disciplina SACI
}

Contributions from initiation to teaching in health training through experiences in outside spaces in the SACI subject

Contribuciones de la iniciación a la docencia en educación para la salud a través de experiencias en espacios extramuros en la disciplina SACI

Jayara Mikarla de Lira

ORCID: https://orcid.org/0000-0002-1707-0983 Universidade Federal do Rio Grande do Norte, Brasil E-mail: jayara-mikarla@hotmail.com

Shirleíze Mariane Pereira Santos

ORCID: https://orcid.org/0000-0002-2652-2515 Universidade Federal do Rio Grande do Norte, Brasil E-mail: shirleize_smps@hotmail.com

Flávia Rayonara Santana da Silva ORCID: https://orcid.org/0000-0001-7004-1199 Universidade Federal do Rio Grande do Norte, Brasil E-mail: rayonara10@hotmail.com

Loorrana Beatriz da Silva Flor

ORCID: https://orcid.org/0000-0003-0022-8016 Universidade Federal do Rio Grande do Norte, Brasil E-mail: loorrannabb@gmail.com

José Jailson de Almeida Júnior

ORCID: https://orcid.org/0000-0001-7448-0703 Universidade Federal do Rio Grande do Norte, Brasil E-mail: jailson.junior@ufrn.br

\begin{abstract}
Resumo
O presente trabalho tem como objetivo analisar as contribuições do ser monitor na disciplina Saúde e Cidadania na formação acadêmica dos futuros profissionais da saúde. Utilizou-se o método de pesquisa bibliográfica nas bases de dados eletrônicas Scielo, Google Scholar e na Biblioteca Virtual da Saúde (BVS) para uma fundamentação teórica sobre a temática. O banco de dados da pesquisa foi composto por duas etapas, primeiro a análise dos relatórios dos monitores da referida disciplina dos anos 2015 e 2016 da Faculdade de Ciências da Saúde do Trairi (FACISA/UFRN), segundo a realização de 4 Grupos Focais entre os meses de maio e junho de 2016, sendo composto por 6 discentes cada. Os grupos focais foram gravados e transcritos na íntegra, originando-se um banco de dados submetido a análise no Software IRAMUTEQ. A exploração dos registros dos relatórios dos monitores da SACI permitiu a construção de uma categoria denominada "Contribuições na formação acadêmica pela vivência na monitoria da SACI". Já o material obtido pelos grupos focais proporcionou a formação de três categorias temáticas, são estas: A compreensão da disciplina SACI; A experiência no ambiente social e serviços de saúde e o (re)aprender no dia a dia. Os pilares estruturais de todas as universidades são: ensino, pesquisa e extensão. No ensino destaca-se a monitoria, instrumento de melhoria do ensino-aprendizagem na graduação por meio da cooperação mútua entre discente monitor, docente e discentes alvos da monitoria, além de agir como um estímulo para a iniciação à docência e para sua valorização.
\end{abstract}

Palavras-chave: Docentes; Ensino; Universidades.

\begin{abstract}
This paper aims to analyze the contributions of being a monitor in the Health and Citizenship discipline in the academic training of future health professionals. The bibliographic research method was used in electronic databases Scielo, Google Scholar and the Virtual Health Library (VHL) for a theoretical foundation on the subject. The research database consisted of two stages, first the analysis of the reports of the monitors of the referred discipline from the years 2015 and 2016 of the Faculty of Health Sciences of Trairi (FACISA/UFRN), second the realization of 4 Focus Groups among the months of May and June 2016, consisting of 6 students each. The focus groups were recorded and transcribed in full, resulting in a database submitted for analysis in the IRAMUTEQ Software. The exploration of the records of the reports of the SACI monitors allowed the construction of a category called "Contributions to academic
\end{abstract}


training through the experience in monitoring SACI". The material obtained by the focus groups provided the formation of three thematic categories, namely: Understanding of the SACI discipline; Experience in the social environment and health services and (re)learning in everyday life. The structural pillars of all universities are: teaching, research and extension. teaching-learning in graduation through mutual cooperation between monitor student, teacher and target students of monitoring, in addition to acting as a stimulus for initiation to teaching and for its appreciation.

Keywords: Faculty; Teaching; Universities.

\section{Resumen}

Este estudio tiene como objetivo analizar las contribuciones de ser monitor en la disciplina de Salud y Ciudadanía en la formación académica de los futuros profesionales de la salud. El método de investigación bibliográfica se utilizó en las bases de datos electrónicas Scielo, Google Scholar y la Biblioteca Virtual en Salud (BVS) para una base teórica sobre el tema. La base de datos de la investigación constó de dos etapas, primero el análisis de los informes de los monitores de la referida disciplina de los años 2015 y 2016 de la Facultad de Ciencias de la Salud de Trairi (FACISA / UFRN), segundo la realización de 4 Focus Groups entre los meses de mayo y junio de 2016, compuesto por 6 alumnos cada uno. Los grupos focales se grabaron y transcribieron en su totalidad, lo que resultó en una base de datos enviada para su análisis en el software IRAMUTEQ. La exploración de los registros de los informes de los monitores de la SACI permitió la construcción de una categoría denominada "Aportes a la formación académica por la experiencia en el seguimiento de la SACI". El material obtenido por los grupos focales permitió la formación de tres categorías temáticas, a saber: Comprensión de la disciplina SACI; Experiencia en el entorno social y servicios de salud y (re) aprendizaje en la vida cotidiana. Los pilares estructurales de todas las universidades son: la docencia, la investigación y la extensión. Destinatarios de la monitorización, además de actuar como estímulo para la iniciación a la docencia y para su apreciación.

Palabras clave: Docentes; Enseñanza; Universidades.

\section{Introdução}

A universidade é um espaço de construção e compartilhamento de conhecimento, onde os discentes têm a sua formação profissional complementada pela vivência em três pilares fundamentais: $O$ ensino, a pesquisa e a extensão. Nesse sentido, vale ressaltar o ensino como importante ferramenta para o fortalecimento do conhecimento aprendido em sala de aula ou em vivências extramuros, destacando-se nesse pilar a vivência na monitoria (Frison, 2016).

A monitoria destaca-se como instrumento para melhoria do ensino-aprendizagem na graduação, além de permitir o estabelecimento de novas práticas e experiências pedagógicas, tendo como finalidade promover a cooperação mútua entre discente monitor, docente e discentes alvos da monitoria.

A Lei No 9.394 (1996) que estabelece sobre as diretrizes e bases da educação nacional, traz que os discentes da educação superior que exercem função de monitoria, também podem realizar atividades de ensino e pesquisa, caso seja condizente com o projeto pedagógico do seu curso. Assim, o monitor tem a oportunidade de agregar saberes e vivências a sua formação, como também, desenvolve uma capacidade crítica-reflexiva.

A Resolução N 569 de 8 de dezembro de 2017, que trata sobre as Diretrizes Curriculares Nacionais (DCN) de todos os cursos de graduação na área da saúde, aponta para a necessidade do compromisso das Instituições de Ensino Superior com a realização das atividades de ensino, pesquisa e extensão. Ainda aborda sobre a comum valorização da pesquisa em detrimento da iniciação à docência, mas que este cenário deve ser reavaliado pelas instituições superiores a fim de alcançar uma maior valorização das atividades de monitoria desenvolvidas pelos discentes, pois seu envolvimento é fundamental para a transformação da formação em saúde em profissionais críticos, colaborativos e consciente do seu papel social (Conselho Nacional de Saúde [CNS], 2017).

Nesse cenário, a monitoria surge como estímulo de iniciação à docência, apontando para um programa de valorização docente, haja vista que os alunos trabalham juntamente com o professor orientador e estabelece meios de permitir um ensino facilitado para os alunos monitorados que participam da atividade de ensino.

Em relação ao vínculo com o conhecimento e as questões educacionais, a monitoria é um espaço de aprendizagem 
proporcionado aos alunos. Sua finalidade é aperfeiçoar o processo de formação profissional e promover a melhoria da qualidade de ensino, criando condições para o aprofundamento teórico e o desenvolvimento de habilidades relacionadas à atividade docente do monitor. Os programas de monitoria têm que proporcionar aos graduandos a possibilidade de otimizar o seu potencial acadêmico, auxiliando-os na formação profissional (Natário \& Santos, 2010).

Dentre as vantagens da experiência, existe o processo de troca mútua do conhecimento entre monitor-monitoradodocente orientador, consolidando um aprendizado efetivo para os envolvidos neste cenário de ensino, além do domínio maior pelo monitor das diversas áreas do conhecimento que são desafiados a trabalhar em um projeto de ensino, contribuindo para sua formação enquanto profissional de saúde (Andrade et al., 2018).

Baseado na relevância da iniciação à docência, a Universidade Federal do Rio Grande do Norte (UFRN) criou o programa de monitoria da disciplina Saúde e Cidadania (SACI), uma disciplina desenvolvida no ambiente social e nos serviços de saúde do Sistema Único de Saúde (SUS). Para tornar-se monitor da disciplina, precisa necessariamente ter cursado essa disciplina em algum momento da sua formação, para que possa ter uma compreensão do funcionamento e modos de contribuir com os monitorados que estão vivenciando pela primeira vez a SACI.

Considerando a relevância do programa de monitoria para uma formação crítica-reflexiva dos futuros profissionais de saúde, como também, as vantagens que ela apresenta para o ambiente acadêmico e social, este trabalho apresenta como objetivo, analisar as contribuições do ser monitor na disciplina SACI na formação acadêmica dos futuros profissionais da saúde.

\section{Metodologia}

O presente trabalho utilizou o método de pesquisa bibliográfica nas bases de dados eletrônicas Scielo, Google Scholar e na Biblioteca Virtual da Saúde (BVS), que tem como objetivo conhecer as diferentes contribuições científicas sobre determinado tema ou fenômeno (Sousa et al., 2021). Na busca adotou-se os critérios de inclusão: artigos que versassem sobre a monitoria na iniciação à docência e a importância desta na formação acadêmica, para uma melhor compreensão da temática.

Como banco de dados, utilizou-se os relatórios dos monitores da disciplina SACI, dos anos 2015 e 2016 da Faculdade de Ciências da Saúde do Trairi (FACISA/UFRN). Os relatórios avaliativos surgem como uma ferramenta de avaliação formativa, com o objetivo de conhecer o processo de construção do conhecimento do estudante ao longo do processo de ensino-aprendizagem (Hoffmann, 2015).

Neste trabalho faz-se uso dos comentários escritos nos relatórios de monitoria feitos pelos discentes monitores, para manter o anonimato e o sigilo as falas dos participantes serão identificadas, no texto, com números do algarismo romano.

Além disso, para o levantamento de dados foi utilizado a estratégia de Grupos Focais, que consiste em uma técnica de coleta de dados qualitativos. Conforme Backes et al. (2011), os grupos focais é caracterizado como uma entrevista em grupo, na qual a interação entre os entrevistados configura-se como parte integrante do método, permitindo uma interação reflexiva sobre determinado tema ou fenômeno.

Foram realizados 4 Grupos Focais entre os meses de maio e junho de 2016, sendo composto por 6 discentes cada. Os Grupos Focais foram gravados e as falas posteriormente transcritas na íntegra. Os entrevistados serão identificados como Indivíduos e classificados por algarismos, a fim de preservar o anonimato.

O material obtido por meio dos Grupos Focais foi transformado em um Corpus, que é criado a partir de um conjunto de "textos" para ser analisado na Interface de R pour les Analyses Multidimensionnelles de Textes et de Questionnaires (IRAMUTEQ), este software permite uma compreensão dos discursos que serão analisados no trabalho, resultando em uma melhor interpretação e compreensão das falas, pois identifica o contexto em que as palavras ocorre, faz análise lexical do 
material e cria classes hierárquica (Salviati, 2017).

A análise do corpus total permitiu observar que ele era constituído por 206 textos, separados em 691 segmentos de texto, com o aproveitamento de 87,84\%. Emergiram 22.151 ocorrências (palavras, formas e vocabulários), sendo que 2.711 são palavras distintas e 1.425 ocorrem uma única vez. O conteúdo analisado foi categorizado, surgindo quatro classes: Classe 1 com 191 segmentos de texto (31,47\%), Classe 2 com 170 (28,01\%), Classe 3 com 76 (12,52\%), Classe 4 com 170 (28,01\%).

As classes 1 e 4 são interligadas, originando o Subcorpus A que trata da vivência na comunidade através da SACI. As classes 2 e 3 também apresentaram uma relação, emergindo a discussão sobre a percepção da disciplina e sua contribuição para a formação em saúde.

\section{Resultados e Discussão}

O material obtido pelos grupos focais e analisado no IRAMUTEQ permitiu a formação de três categorias temáticas, são estas: "A compreensão da disciplina SACI"; "A experiência no ambiente social e serviços de saúde" e o "(Re)aprender no dia a dia". Enquanto a exploração dos registros dos relatórios dos monitores da SACI permitiu a construção de uma categoria denominada "Contribuições na formação acadêmica pela vivência na monitoria da SACI".

\subsection{A compreensão da disciplina SACI}

A formação em saúde vem sendo debatida desde a Reforma Sanitária (1970) e os movimentos populares na saúde, tendo em vista a hegemonia do conhecimento técnico-prático no cuidado em saúde, em detrimento aos condicionantes e determinantes da saúde (Paiva \& Teixeira, 2014).

Nesse cenário, a inclusão de disciplinas voltadas para o meio social e o ambiente dos serviços de saúde, surgem como potencial na formação dos profissionais de saúde para o estímulo a um olhar mais sensível à saúde em seu contexto sociocultural, não limitando o ser individual ou coletivo ao seu aspecto biológico. O que pode ser observado nas falas abaixo:

Indivíduo 8: [...] ]eu tenho colegas que não pagaram as disciplinas de humanas, que não pagaram saci e os que pagaram saci que se comprometeram, participaram, ajudaram e o atendimento é muito melhor. Eles enxergam como um todo, completo a pessoa, não a doença [...]

Assim, nota-se que a disciplina SACI atua como um diferencial na formação de um novo perfil de profissional, na medida em que possibilita um primeiro contato com a comunidade, onde os estudantes dos diferentes cursos vão se notar diante de várias problemáticas e através de uma reflexão crítica desenvolvem métodos de resolutividade da situação que surgem ao longo da vivência.

Dessa forma, o espaço extramuros torna-se um ambiente de aprendizado diverso, que exige do aluno uma flexibilidade ao desenvolver suas experiências. A experiência extramuros permite que a formação em saúde seja baseada em uma reflexão sobre o seu processo de aprendizado e passem a ter uma nova visão sobre o fazer saúde e é justamente neste ponto que essas experiências são acrescentadas de um olhar mais sensível, rompe-se assim com o modelo tradicional no qual o homem é transformado em um objeto (Maciel et al., 2005).

Conforme Biscarde et al. (2014) "a vivência extensionista revela-se fundamental na formação universitária, propiciando experiências ampliadas aos graduandos, muito além daquelas obtidas nos moldes tradicionais e bancários de formação profissional". Desse modo, esse contato com a realidade e com o funcionamento da assistência marcam a formação universitária dos graduandos, evidenciado nesta fala: 
Indivíduo 3:[...]A gente também saiu andando praticamente casa por casa, a gente foi a pé, e ele (agente comunitário de saúde) conhecia realmente todo mundo[...]

O ensino tradicional promove uma formação com base na transmissão do conhecimento, valorização do técnico e científico, desconexão dos temas, carga horária excessivas para determinados conteúdos, além do ensino ser abreviado a sala de aula. Desse modo, a disciplina SACI que é caracterizada como uma vivência nos espaços extramuros amplia as condições para que o estudante, de forma ativa, crie o seu próprio conhecimento e desenvolva uma capacidade crítica-reflexiva sendo essas aberturas fundamentais para uma formação integradora (Biscarde et al., 2014).

A aprendizagem pautada na autonomia, protagonismo e desenvolvimento do lúdico perpassa pela mudança de paradigmas em relação as metodologias de ensino, com o objetivo de alcança profissionais capazes de desenvolver um cuidado humano e sensível. Nesse cenário, precisa-se de espaços que contemple os discentes em sua formação científica, política, social e subjetiva para que os mesmos possam contextualizar os conhecimentos aprendidos e experienciar esses novos modos de formação (Silva \& Sei, 2021; Silva et al., 2020).

Ao adentrar na comunidade os estudantes estão rompendo as fronteiras da universidade, ao mesmo tempo que formam pontes que geram fluxos de conhecimento, é uma aprendizagem viva, aprender a ser junto com o outro e não somente aprender porque o conhecimento está sendo depositado nas aulas, é uma experimentação do que é ser um promotor de saúde, tornandose seu cuidado diferenciado, pois foi experimentando o cotidiano e uma nova forma de ver o outro em sua subjetividade e necessidades.

Ademais, a SACI promove a adoção por parte dos graduando do seu compromisso enquanto futuros profissionais da saúde na implementação de mudanças relacionadas aos princípios do SUS, sendo uma das principais, despertar nas pessoas o interesse por seu direito à saúde, para que assim sintam-se empoderadas e busque a transformação da realidade, haja vista a importância da participação popular para a garantia da eficiência do SUS, sendo essa uma conquista do povo, compreende-se assim que o povo é fundamental para o seu desenvolvimento (Pinheiro et al., 2020).

\subsection{A experiência no ambiente social e serviços de saúde}

A imersão dos graduandos no cotidiano das pessoas e da atenção primária à saúde, possibilita um espaço rico para a construção do aprender a aprender e, assim, aprender a ser e a cuidar. Opostamente, ao ensino tradicional resume-se a prática do cuidado em um ambiente recortado que é os hospitais universitários e com o foco somente em processos patológicos (Garcia et al, 2021).

Na comunidade os discentes vão conhecer e aprender sobre pessoas, culturas e modo de vidas diferentes, que não é possível em um local fechado como os hospitais, percebe-se assim a importância da vivência na comunidade (Biscarde et al., 2014; Souza et al.,2019). Essa desconstrução do modelo biomédico ocorre por meio das experiências ocasionadas nessa vivência:

Indivíduo_4: [...]eu tive vivências a respeito só do processo saúde - doença, sobre isso eu vejo que saci me deu muitas oportunidades, em relação a isso foi um crescimento muito grande e também houve criação de vínculos. Até hoje tem pessoas que agora fazem parte do meu convívio que são da comunidade[...]

A abertura para o aprendizado na comunidade é marcada como uma formação voltada para a importância do indivíduo e sua família no processo saúde-doença, com a valorização da realidade social como um determinante do bem-estar seja 
individual ou coletivamente.

Nessa perspectiva, a importância de uma educação que promova condições para que os discentes desenvolvam atitudes crítica-reflexivas ao conhecer a realidade e assim possam se comprometer com a transformação social é indiscutivelmente necessário. Se o homem não tomar consciência da realidade, ele não participa ativamente da sociedade, da transformação social, pois não terá conhecimento da sua capacidade de transformá-la (Soares, 2020).

Assim, a SACI é reconhecida pelos monitores como uma oportunidade de ouvir o outro, conhecer o trabalho dos profissionais da saúde, além disso da superação de estigmas sociais, como fica evidente nos discursos a seguir:

Indivíduo 4: Eu acredito que a maior ação do nosso grupo foi ouvir as pessoas, a princípio foi o que nós fizemos né, nós estávamos com dificuldade de ir até a casa das pessoas e conversar com elas, até por que elas ficavam meio que desconfiadas, assim não se sentindo tão bem[...]

Desse modo, é primordial a formação de profissionais além da capacitação técnica, que também é importante, mas o homem não se resume apenas a partes fragmentadas, sendo notório que suas relações e interações podem interferir na sua saúde.

\section{$3.3 \mathrm{O}$ (re)aprender no dia a dia}

Os profissionais de saúde vivenciam diariamente um processo de (re) construção de saberes e práticas, para assim buscar oferecer o melhor no cuidado ao seu paciente. Para isso, é necessário desde a universidade que esses profissionais em formação tenham abertura para aprender mais sobre as coisas e pessoas (Souza et al., 2019).

Nesse cenário, o desenvolvimento de uma visão diferenciada, de um olhar humanizado, através desta experiência na comunidade é mais um dos benefícios desta relação:

Indivíduo 6: [...]depois que você vai pra prática clínica, de atender, de tratar e tudo mais, você vê o quanto é importante saci, mas no começo as vezes a conduta não está dando certo e isso é porque você não enxerga o paciente como deveria enxergar[...]

A prática da integralidade que é um dos princípios do SUS é levantado pelos estudantes, no momento em que passam a construir este olhar integral do homem, ressaltando a importância das suas práticas cotidianas, da compreensão do paciente enquanto participante de contextos familiares, sociais e culturais particulares (Carnut, 2017).

Além disso, os monitores apontam para o processo de desconstrução de estigmas e preconceitos que carregavam consigo antes de adentrar a comunidade, como no discurso abaixo:

Indivíduo_4 [...]no início a gente tinha medo. Pensava: é muito longe, é perigoso. É importante falar da nossa desconstrução, a gente chegou lá com muito medo[...]

A SACI contribui para os discentes (re)construam suas práticas relacionadas à assistência aprendidas anteriormente a essa vivência em comunidade, relacionando-os agora com a sua habilidade crítica-reflexiva surgida nas situações da percepção social da realidade, caracterizando a disciplina como uma potencializadora na construção de sujeitos sociais, haja vista a transformação gerada durante a SACI na visão de mundo e na maneira de percepção do fazer saúde (Biscarde et al., 2014; 
Chirelli,\& Sordi, 2021).

\subsection{Contribuições na formação acadêmica pela vivência na monitoria da SACI}

Inicialmente os discentes monitores revelam através dos seus escritos que a experiência como monitor trouxe acréscimos a sua formação, desde a forma de se comportar enquanto ser cidadão, a percepção da necessidade do trabalho em equipe e do dialogar com as pessoas da comunidade.

IV-O programa (de monitoria) tem contribuído na minha formação, permitindo-me refletir sobre os princípios $e$ valores, que orientam o aprimoramento do comportamento ético e cidadão, no relacionamento com colegas da equipe e com as pessoas da comunidade.

A experiência como monitor é carregada de momentos que irão contribuir tanto para sua formação pessoal quanto profissional, pois a flexibilidade ao lidar com o outro, o ouvir e o perceber o diferente enquanto diferente e não como inimigo, serão trabalhadas na vivência a iniciação à docência.

XII- [...] a monitoria ainda proporciona o primeiro passo para aqueles profissionais que se interessam na área da docência, sendo uma experiência muito enriquecedora para dar início a essa caminhada.

VII- A monitoria é um grande estímulo à iniciação à docência. Digamos que o seu orientador (docente) é onde você deve se espelhar para que tenha mais objetivos futuros que queira seguir a área da docência [...]

XI- [...] a inserção dos monitores [...] em conjunto com os docentes da FACISA enriquecem sua experiência como discente e possibilita o ganho de experiência para um futuro caminho da docência [...].

A monitoria é uma forma dos monitores se aprofundar nos conhecimentos e transmiti-los para outros alunos, além da aproximação com a área da docência, seja por possibilitar uma maior comunicação com o docente ou, ou, pela identificação das atividades desempenhadas na monitoria, conduzindo para a iniciação à docência (Santos \& Batista, 2015; Melo, (2017).

O contato permitido pela monitoria com diferentes alunos dos cursos de graduação e comunidades viabiliza aos discentes reflexões críticas e discussões sobre problemas observados, troca de conhecimentos entre discentes e comunidade e uma a busca de melhor qualidade de vida para a população (Pereira, 2018; Rios et al., 2019). Nesse sentido, os estudantes relataram a relevância da monitoria para sua formação pessoal e profissional, como pode ser observado nas falas a seguir:

VII- [...] A monitoria nos dá oportunidade de aprofundar sobre os assuntos da disciplina para buscar mais conhecimentos e habilidades e atitudes técnico-pedagógicas.

VI- Considero a participação no projeto de monitoria na disciplina Saúde e Cidadania de suma importância para a minha formação e para a minha qualificação profissional [...]

XI-[...] a inserção dos monitores no planejamento, desenvolvimento e avaliação das atividades desenvolvidas, como também no processo de capacitação [...] proporcionando a oportunidade do aluno se integrar na pesquisa, na revisão de textos e o ganho de habilidades para o exercício de atividades de educação em saúde, crescimento pessoal e o ganho de sabedoria será com certeza de grande valia para meu futuro profissional na docência.

A experiência extramuros proporciona sair do ambiente da sala de aula e conhecer novas realidades em saúde. Além 
disso, o desenvolvimento de outras habilidades além de técnicas, é fundamental para que os futuros profissionais da saúde tenham um olhar mais cauteloso para o paciente, mais sensível e humano (Lira et al., 2020). Nas falas seguintes os discentes ressaltam as vivências de novas experiências:

I- [...] a experiência como monitora e especialmente dessa disciplina foi de grande valia, pois aprendi lições que levarei para a vida pessoal e profissional, superei desafios e com isso cresci, não só compartilhei conhecimentos como também tive a oportunidade de vivenciar novas experiências e assim enriquecer ainda mais a minha formação acadêmica.

II- Vivi novas experiências e responsabilidades que são importantes para minha formação.

III- O projeto de monitoria foi muito enriquecedor para a minha formação acadêmica pois tive a oportunidade de viver várias experiências construtivas e enriquecedoras.

É importante ressaltar que a prática da monitoria representa um grande desafio, por ser uma experiência nova, exigindo dos discentes uma postura mais séria para lidar com situações problemas que possam surgir e desenvolver formas para ajudar os demais alunos nas suas dúvidas.

Nos relatos dos alunos é possível notar que grande maioria pôde rever seu papel, valorizando a relação com seus docentes possibilitando aos estudantes incentivos por conhecimentos, oportunidades de vivenciar novas experiências como uma futura inserção na docência, na graduação e nota-se a importância da monitoria na iniciação à docência (Nascimento et al, 2021). Podemos ver nos relatos abaixo:

V-[...] Portanto, ao observar a estudante que entrou na monitoria e aquela que está saindo pode-se encontrar uma diferença, pois, existe agora à amplificação do querer cuidar, lutar e exercer efetivamente a cidadania, e o fazer ciência.

\section{Considerações Finais}

Após a análise feita nos relatórios dos monitores da disciplina SACI (Saúde e Cidadania) da UFRN/FACISA e a revisão de literatura, podemos concluir que a monitoria é um instrumento importante na preparação do discente para um futuro docente, que é fundamental no seu processo de aprendizagem e na condução a um futuro profissional voltado para a iniciação à docência.

Além disso, foi possível perceber a importância da experiência extramuros para oferecer ao graduando este contato como comunidade, possibilitando a criação de uma pensamento crítico-reflexivo, com base nas vivências oferecidas pela disciplina SACI. Assim, os estudantes passaram a olhar para o SUS de outra forma, valorizando os seus princípios como a integralidade e a participação popular para o alcance de um SUS eficiente, (des)construindo tanto a visão do próprio SUS, quanto da disciplina, visão está antes desancorada da realidade.

A monitoria contribui para uma maior obtenção e aprofundamento de conhecimentos de áreas específicas, e também para o crescimento pessoal e profissional dos discentes monitores. Oportuniza aos alunos o primeiro contato com a profissão e o desenvolvimento de habilidades da docência, permitindo o contato direto com outros discentes, retirada de dúvidas dos conteúdos vistos em sala de aula possibilitando um contato direto com a comunidade onde estão inseridos, o que acarreta um amadurecimento e crescimento pessoal, acadêmico e profissional.

Foi possível analisar que a monitoria é um programa que ajuda a esclarecer aos discentes o papel, função, 
dificuldades, benefícios e um contato real com a prática, exercendo melhor seu trabalho para uma futura carreira docente.

Como sugestões para trabalhos futuros pode-se realizar novos estudos com profissionais da saúde atuantes que vivenciaram experiências extramuros nas disciplinas da graduação e analisar como é a sua compreensão sobre a influência dessas vivências na sua prática profissional, além disso se a participação em programas de ensino levou a escolha da atuação como docentes universitários.

\section{Referências}

Andrade, E. G. R. D., Rodrigues, I. L. A., Nogueira, L. M. V. \& Souza, D. F. D. (2018). Contribution of academic tutoring for the teaching-learning process in Nursing undergraduate studies. Revista Brasileira de Enfermagem, 71, 1596-603.

Backes, D. S., Colomé, J. S., Erdmann, R. H. \& Lunardi, V. L. (2011). Grupo focal como técnica de coleta e análise de dados em pesquisas qualitativas. $O$ mundo da saúde, 35(4), 438-42.

Biscarde, D. G. D. S., Pereira-Santos, M \& Silva, L.B. (2014). Formação em saúde, extensão universitária e Sistema Único de Saúde (SUS): conexões necessárias entre conhecimento e intervenção centradas na realidade e repercussões no processo formativo. Interface-Comunicação, Saúde, Educação, 18, 177-86.

Carnut, L. (2017). Cuidado, integralidade e atenção primária: articulação essencial para refletir sobre o setor saúde no Brasil. Saúde em Debate, 41, 11771186

Chirelli, M. Q. \& Sordi, M. R. L. D. (2021). Pensamento crítico na formação do enfermeiro: a avaliação na área de competência Educação na Saúde. Revista Brasileira de Enfermagem, 74 (5), 1-9.

Conselho Nacional de Saúde. (2017). Resolução nº 569, de 8 de dezembro de 2017. Diário Oficial da União.

Frison, L. M. B. (2016). Monitoria: uma modalidade de ensino que potencializa a aprendizagem colaborativa e autorregulada. Proposições, $27,133-153$.

Garcia, A., Dariz, W. F., Tonietto, R., Torezan, G. \& Ramon, M. E. G. (2021). Utilização de metodologias ativas no processo de ensino-aprendizagem em saúde. In Congresso movimentos docentes IV sepad e II pratic, 1, 44-48.

Hoffmann, J. (2012). Avaliação e Educação Infantil: um olhar sensível e reflexivo sobre a criança. Porto Alegre: Mediação, 18.

Lira, J. M., Silva, F. R. S., Vieira, H. W. D., Fernandes, M. N. F. \& Almeida Júnior, J. J. (2020). A experiência do aluno na educação em saúde em espaços universitários extramuros. Research, Society and Development, 9 (11), 1-19.

Lei n 9.394, de 20 de dezembro de 1996 (1996). Estabelece as diretrizes e bases da educação nacional. Diário Oficial da União. Brasília, DF.

Maciel, R. V., Silva, P. T. G., Sampaio, R. F. \& Drummond, A. F. (2005). Teoria, prática e realidade social: uma perspectiva integrada para o ensino de fisioterapia. Fisioterapia em Movimento, 18(1).

Melo, G. F. (2017). Monitoria: projeto formativo para iniciação à Docência Universitária. Revista Eletrônica Pesquiseduca, 9(17), 57-71.

Nascimento, J. T., Silva Cardoso, L. T., Araújo, L. C. N., Nascimento Oliveira, V. V., Santos Silva, E., Silva, P. J. T. G. \& Clemente, J. W. F. S. (2021). Monitoria como espaço de iniciação à docência. Revista Eletrônica Acervo Saúde, 13(2), e5577-e5577.

Natário, E. G. \& Santos, A. A. A. D. (2010). Programa de monitores para o ensino superior. Estudos de Psicologia (Campinas), $27,355-364$.

Paiva, C. H. A. \& Teixeira, L. A. (2014). Reforma sanitária e a criação do Sistema Único de Saúde: notas sobre contextos e autores. História, Ciências, SaúdeManguinhos, 21, 15-36.

Pereira, M. F. (2018). Interprofissionalidade e saúde: conexões e fronteiras em transformação. Interface-Comunicação, Saúde, Educação, $22,1753-1756$.

Pinheiro, F. T. D. S., Tavares, N. B. F., Silva, J. P. X. \& Araújo, A. F. D. (2020). Participação universitária em defesa do Sistema Único de Saúde. Saúde em Debate, 44, 504-515.

Rios, D. R. D. S., Sousa, D. A. B. D. \& Caputo, M. C. (2019). Diálogos interprofissionais e interdisciplinares na prática extensionista: o caminho para a inserção do conceito ampliado de saúde na formação acadêmica. Interface-Comunicação, Saúde, Educação, 23.

Salviati, M. E. (2017). Manual do Aplicativo Iramuteq (versão 0.7 Alpha 2 e R Versão 3.2.3) [Manual]. Planaltina, DF.

Santos, G. M. \& Batista, S.H.S.S. (2015). Monitoria acadêmica em formação em/para saúde: desafios e possibilidades no âmbito de um currículo interprofissional em saúde. ABCS Health Sciences. 40 (3), 203-207.

Silva, A. C. de M. \& Sei, M. B. (2021). A Humanização na Formação Acadêmica em Saúde: Perspectiva de Egressos de um Projeto de Extensão. Revista Psicologia e Saúde, 13(3), 3-18.

Silva, R. P., Camacho, A. C. L. F., Silva, M. A. P. \& Menezes, H. F. (2020). Strategies for the use of active methodology in the training of nursing academics: experience report. Research, Society and Development, 9(6), 1-11. 
Research, Society and Development, v. 11, n. 1, e31111124789, 2022

(CC BY 4.0) | ISSN 2525-3409 | DOI: http://dx.doi.org/10.33448/rsd-v11i1.24789

Soares, R. O. (2020). A construção histórica do sujeito nas obras de Paulo Freire. História Revista, 25(3), $191-206$.

Sousa, A. S., Oliveira, G.S. \& Alves, L.H. (2021). A pesquisa bibliográfica: princípios e fundamentos. Cadernos da FUCAMP, 20(43).

Souza, E. C. D., Castro Júnior, A. R. D., Cavalcante, A. S. P., Torres, R. A. M. \& Silva, M. R. F. D. (2019). Projeto Vivências e Estágios na Realidade do Sistema Único de Saúde: linha de fuga na formação em saúde para uma atuação na saúde coletiva. Saúde em Debate, 43, 897-905. 